\title{
MULTIWAVELENGTH STUDIES OF $\beta$ CEPHEI STARS
}

\author{
H. CUGIER ${ }^{1}$, A. PIGULSKI ${ }^{1}$, G. POLUBEK ${ }^{1}$ and R. MONIER ${ }^{2}$ \\ ${ }^{1}$ Astronomical Institute, Wroclaw University, Kopernika 11, 51-622 Wroclaw, Poland \\ ${ }^{2}$ IUE Observatoty VILSPA, P.O. Box-Apartado 50727, Madrid 28080, Spain
}

As first pointed out by Moskalik and Dziembowski (1992) all $\beta$ Cephei stars lie within the domain of $\mathrm{H}-\mathrm{R}$ diagram where $\kappa$-mechanism effectively drives pulsations in the stellar layers with $T \approx 2 \times 10^{5} \mathrm{~K}$. For most of these objects a chemical composition described by $\mathrm{X}=0.70$ and $\mathrm{Z}=0.02$ is sufficient to account for the pulsations, cf. Dziembowski and Pamyatnykh (1993). Recently, Cugier, Dziembowski and Pamyatnykh (1993) have investigated how the present knowledge about nonadiabatic observables of $\beta$ Cephei stars affects methods of identification of the spherical harmonic degree, $l$. They found that good photometric and radial velocity data should result in unambiguous identification of $l$. Cugier, Dziembowski and Pamyatnykh also concluded that nonadiabatic observables can be used to obtain mean stellar parameters of pulsating stars.

We report here, as examples, the studies of $\delta$ Ceti and BW Vulpeculae. The above mentioned analysis of the ground-based photometric data of $\delta$ Cet taken from Jerzykiewicz et al. (1988) indicates: $l=0, p_{2}, \log T_{\text {eff }}=4.346$ and $\log g=3.73$. Figure 1 shows that indeed only a model with $l=0$ is able to explain the observed flux behaviour of $\delta$ Cet in the satellite ultraviolet region. Futhermore, the observed phases of flux maximum as a function of wavelength offer the possibility to determine the effective temperature of $\beta$ Cephei stars with high precision as Fig. 2 shows for $\delta$ Cet.

In Fig. 3 the observed light ranges for BW Vul are compared with the nonadiabatic model $\left(l=0, p_{1}, \log T_{\text {eff }}=4.29\right.$ and $\left.\log g=3.71\right)$. As one can see, a very good agreement exists even for this star, which is rather extreme case among $\beta$ Cephei stars considering its large light and radial-velocity amplitudes.

\section{Acknowledgements}

This work was supported by the research grant No. 212419104 from the Polish Scientific Research Committee (KBN).

\section{References}

Cugier, H. Dziembowski, W.A. and Pamyatnykh, A.A.: 1993, this issue Dziembowski, W.A. and Pamyatnykh, A.A.: 1993, MNRAS 262, 204

Jerzykiewicz, M., Sterken, C. and Kubiak, M.: 1988, A\&SAS 72, 449

Moskalik, P. and Dziembowski, W.A.: 1991, $A \& A$ 256, L5 


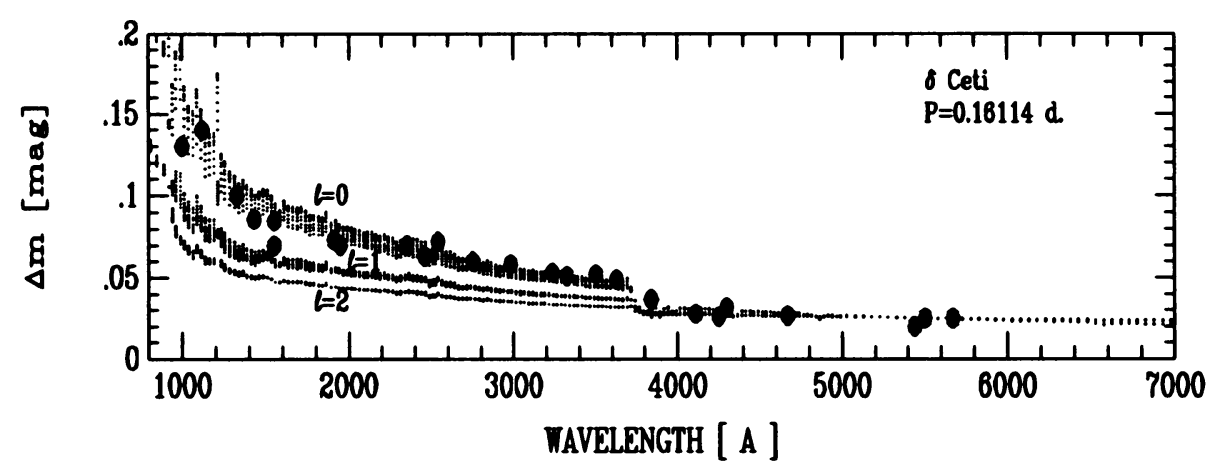

Fig. 1. Calculated light ranges $\Delta m$ (dotted lines) in comparison with UV and visual observations (filled circles) for $\delta$ Cet. All nonadiabatic models with $l=0,1$ and 2 have the same period $(P=0.16114 \mathrm{~d})$

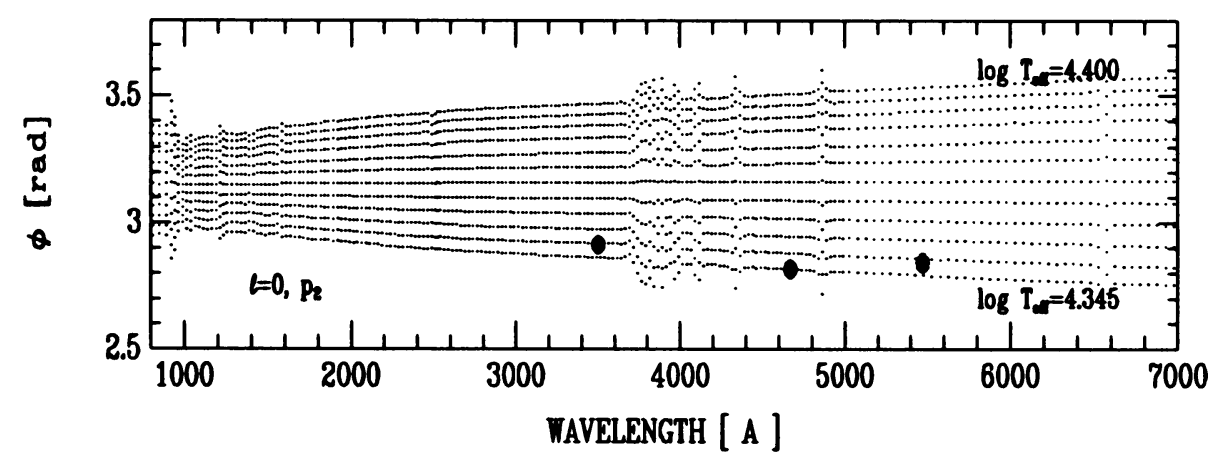

Fig. 2. The observed (filled circles with error bars) phases of flux maximum for $\delta$ Cet are plotted together with nonadiabatic calculations (dots) corresponding to $p_{2}$ mode of $l=0$. Stellar models (all with the period equal to $0.16114 \mathrm{~d}$ ) are labelled by $\log T_{\text {eff }}$ values. The step in $\log T_{\text {eff }}$ is equal to 0.005 dex.

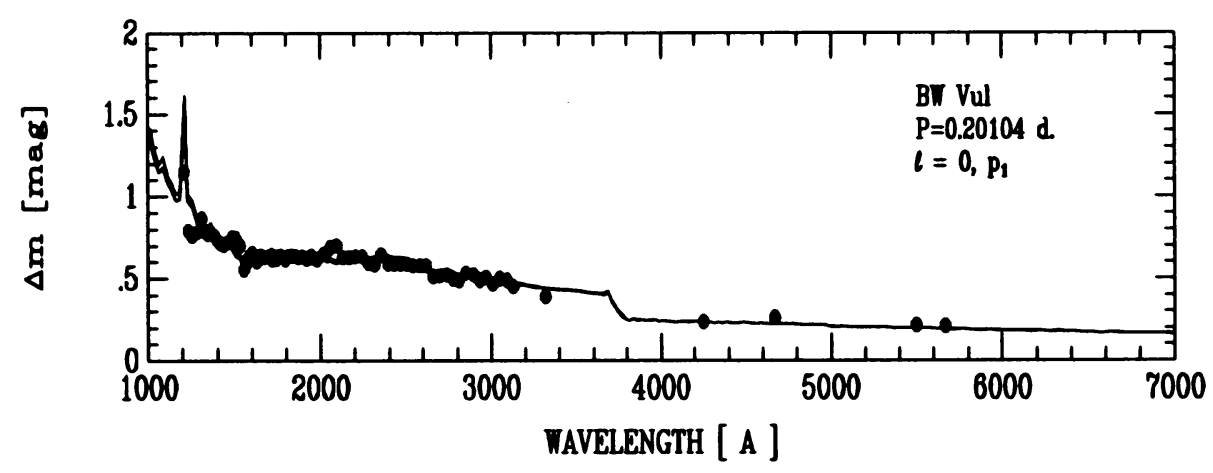

Fig. 3. The best-fit nonadiabatic model compared with the IUE observations of BW Vul. 